\title{
NextStep: Um Protótipo para o Sequenciamento Inteligente e Adaptativo de Enunciados em Programação de Computadores
}

\author{
Carolina Moreira ${ }^{1,2}$, Andrey Ricardo Pimentel ${ }^{1}$, Eleandro Maschio ${ }^{2}$ \\ ${ }^{1}$ Programa de Pós-Graduação em Informática \\ Universidade Federal do Paraná (UFPR) \\ Curitiba - PR - Brasil. \\ ${ }^{2}$ Coordenação do Curso de Tecnologia em Sistemas para Internet \\ Universidade Tecnológica Federal do Paraná (UTFPR) \\ Guarapuava - PR - Brasil. \\ \{cmoliveira, andrey\}@inf.ufpr.br, eleandromeutfpr.edu.br
}

\begin{abstract}
This article details the results with intelligent and adaptive exercise ordering, considering the student dynamic modeling in Computer Programming domain. The methodology uses genetic graphs as a basis for the internal representation. Thus, overlaying aspects highlight the progress of the learner compared to the domain knowledge, and to the individual contribution of each exercise.
\end{abstract}

Resumo. O presente artigo detalha os resultados obtidos com um sequenciamento inteligente e adaptativo de enunciados, considerando a modelagem dinâmica do aprendiz, no domínio de Programação de Computadores. A metodologia utiliza grafos genéticos como base para a representação interna. Com isso, aspectos de sobreposição destacam o progresso do aprendiz frente ao conhecimento do domínio, além da contribuição individual de cada enunciado.

\section{Introdução}

O aprimoramento do processo de ensino-aprendizagem nas disciplinas de Programação de Computadores tem se mantido como um grande desafio [Norvig 2001] por décadas. $O$ grau de dificuldade aumenta nos níveis iniciais, principalmente por se tratar de conhecimentos que são base para qualquer curso na área da Computação. Assim, é importante encontrar meios de diminuir o impacto nesses níveis, bem como atenuar os altos índices de reprovações e de desistências nos respectivos cursos [Miliszewska and Tan 2007].

Dentre as várias abordagens para apoiar o processo de ensino, destacaram-se os Sistemas Tutores inteligentes (STIs). Tratam-se de sistemas projetados para associar técnicas de Inteligência Artificial, de maneira que saibam como o que ensinar, a quem ensinar e como ensinar [Nwana 1990]. Assim, objetivam maior aproximação das aulas individuais, considerando características particulares do aprendiz para variar a interação.

Nesse sentido, a modelagem do aprendiz responsabiliza-se por armazenar o estágio atual de conhecimento e o desempenho do indivíduo sobre os tópicos tutorados. A partir dela, o sistema consegue definir o nível de dificuldade relativo de um conteúdo frente ao conhecimento do aprendiz, além de propor estratégias de tutoria personalizadas. 
Paralelamente, qualquer ambiente de ensino que utilize enunciados precisa, de alguma maneira, determinar a sequência em que eles serão oferecidos ao aprendiz. Alguns sistemas apresentam os enunciados em ordem predefinida [Weber and Brusilovsky 2001]. Outros, permitem que os aprendizes selecionem o próximo enunciado a partir de uma lista estática de alternativas [Weber and Mollenberg 1995]. Em ambas as situações, a escolha do enunciado não considera as capacidades atuais do aprendiz.

Levando em consideração a modelagem do aprendiz, um STI tem a capacidade de sugerir enunciados próprios ao nível de conhecimento do indivíduo. Isso se mostra positivo, pois enunciados com dificuldade muito abaixo desse nível podem causar entediamento; e, do contrário, sendo muito acima, provocar desmotivação [Pimentel and Direne 1998]. Ambos os casos são passíveis do abandono da atividade proposta.

Dessa forma, existe espaço de contribuição para abordagens de sequenciamento adaptativas e inteligentes de enunciados, buscando auxiliar diferentes perfis de aprendizes, independentemente dos níveis de conhecimento. Com isso, pode-se diversificar a ordem e mesmo a quantidade de enunciados e potencializar o processo de ensino-aprendizagem para cada aprendiz em particular.

\section{Contextualização da Solução Proposta}

O objetivo geral desta pesquisa foi propor uma abordagem para o sequenciamento adaptativo de enunciados, frente a uma modelagem dinâmica do aprendiz, no contexto de Programação de Computadores. Aspectos de resenha literária foram abrangidos na publicação de resultados parciais à comunidade [Moreira et al. 2015].

A metodologia de sequenciamento proposta foi implementada no protótipo de ferramenta denominado NextStep, que adota grafos genéticos [Goldstein 1979] como base para a representação interna e externa. Assim, aspectos de sobreposição no grafo destacam o progresso do aprendiz frente ao conhecimento do domínio, além da contribuição individual de cada enunciado. Portanto, no contexto do grafo genético, a sobreposição enfatiza que a estrutura correspondente ao Modelo do Aprendiz deriva do conhecimento do domínio, sendo um subgrafo (ou subconjunto) dele.

Partindo dos modelos do aprendiz e do domínio, proveu-se um processo de ordenação heurística que orienta conteúdos a serem explorados pelo aprendiz. Indicam-se perícias que deveriam ser prioritariamente desenvolvidas (compreendidas e exercitadas) pelo indivíduo, conforme o seu nível atual de conhecimento. Depois, são sugeridos enunciados que contemplem o desenvolvimento dessas perícias.

Alguns passos metodológicos, nesse sentido, foram antecipados pela pesquisa de [Maschio 2013]. O mesmo estudo desenvolveu duas ferramentas que atuam nas primeiras etapas do processo. A primeira ferramenta se concentra na descrição do conhecimento do domínio por meio do detalhamento das perícias componentes. A segunda destinase à catalogação de enunciados descritos como subgrafos do conhecimento de domínio formalizado pela primeira.

O protótipo desenvolvido pela presente pesquisa utiliza dos recursos providos por essas duas ferramentas. Implementa-se uma modelagem dinâmica do aprendiz, assumindo a representação interna e externa por grafos genéticos. Então se utiliza de uma 
V Congresso Brasileiro de Informática na Educação (CBIE 2016)

Anais dos Workshops do V Congresso Brasileiro de Informática na Educação (CBIE 2016)

ordenação heurística para indicar quais perícias devem ser prioritariamente desenvolvidas pelo aprendiz para, em seguida, apresentar os enunciados que contemplem essa demanda.

\section{Dinâmica de Funcionamento da Solução Proposta}

A dinâmica da solução proposta é contextualizada no domínio de Programação de Computadores. O cenário de utilização simula e acompanha o processo de sequenciamento de enunciados para um aprendiz. O processo é divido nas etapas de: (1) situar o aprendiz; (2) sugerir perícia; (3) sugerir enunciado; e (4) avaliar solução. No exemplo, foi suposta a aplicação do processo para um aprendiz que teve apenas uma introdução teórica aos primeiros tópicos da área. A explicação tem caráter resumido, procurando enfoque na fluência desse processo.

O Modelo do Aprendiz é um subconjunto nulo do conhecimento do domínio, pois nenhuma perícia do grafo genético foi desenvolvida ainda. A ferramenta, em primeiro momento, situa o aprendiz frente ao conhecimento do domínio. Faz-se isso como amparo metacognitivo, por meio de representações externas, de caráter visual (sobreposição no grafo genético) e textual (painel de estatísticas), como pode ser observado na Figura 1.

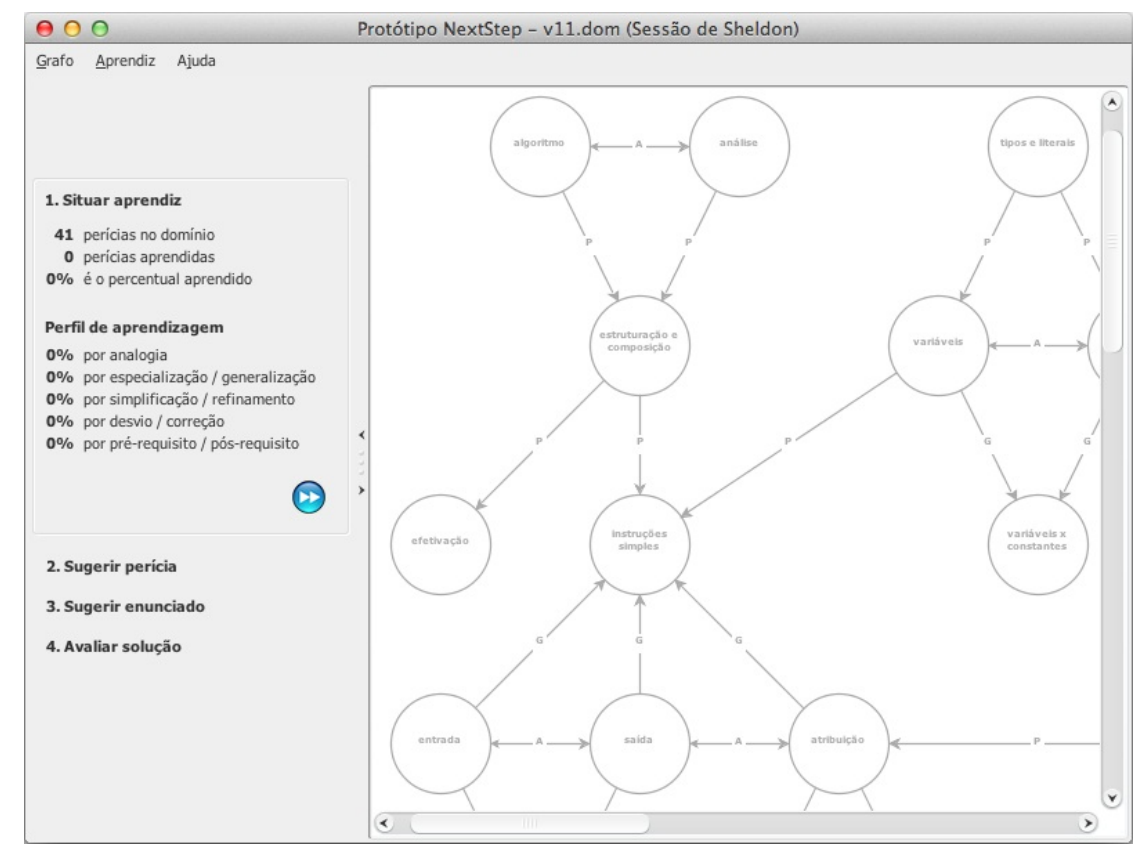

Figura 1. Protótipo NextStep: Situar aprendiz

A ferramenta sugere exclusivamente a perícia inicial do domínio, que possui o identificador algoritmo, para ser desenvolvida na sessão de sequenciamento. Admitindo a perícia como prioritária a ser exercitada, cabe à ferramenta encontrar enunciados que contribuam para esse desenvolvimento. Para isso, toma-se o catálogo de enunciados assumido integralmente de [Maschio 2013].

De todo o catálogo, apenas os enunciados Olá mundo, Olá usuário e Metade de um real contribuem para a apropriação da perícia algoritmo. Convém destacar que, embora todos os enunciados de um catálogo envolvam, por exemplo, instruções de saída, nem todos se propõem a desenvolver esse conhecimento. 
V Congresso Brasileiro de Informática na Educação (CBIE 2016)

Anais dos Workshops do V Congresso Brasileiro de Informática na Educação (CBIE 2016)

Como se tem mais de um enunciado que contempla a perícia sugerida, oferece-se navegabilidade ao aprendiz. A escolha é orientada pela ordem das sugestões, priorizando os enunciados que abranjam o menor número de perícias não desenvolvidas e, depois, aqueles que exercitam o menor número de perícias ao todo (mesmo que já tenham sido desenvolvidas). Essas duas informações são apresentadas ao lado do identificador de cada enunciado. Adicionalmente, quando se seleciona um enunciado, as perícias que ele objetiva desenvolver (regiões do grafo) são destacadas no conhecimento do domínio (Figura 2).

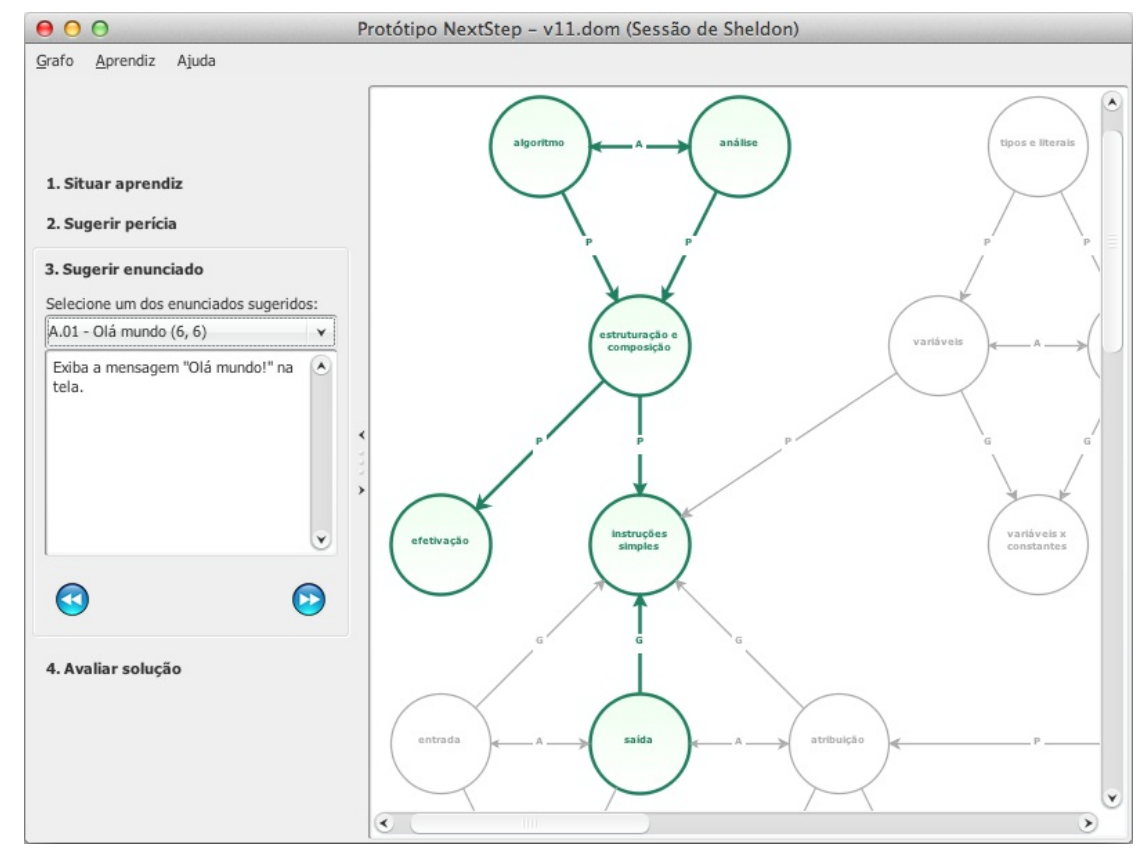

Figura 2. Protótipo NextStep: Sugerir enunciado

Então, supondo-se que o primeiro enunciado (Olá mundo) tenha sido a escolha do aprendiz para exercitar a perícia algoritmo e que a resolução seja avaliada como correta na ferramenta. Depois disso, o Modelo do Aprendiz é atualizado de modo que a refletir que as perícias exercitadas foram desenvolvidas. Exibe-se um sumário dessa atualização, indicando quais outras perícias foram desenvolvidas no processo e que o ciclo de sequenciamento foi completado (Figura 3).

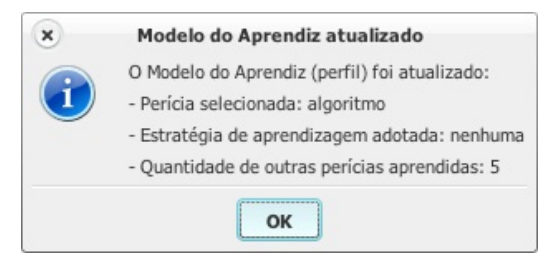

Figura 3. Protótipo NextStep: Atualizar Modelo do Aprendiz

Considerando o Modelo do Aprendiz atualizado, cabe à ordenação heurística da ferramenta sugerir a próxima perícia a ser desenvolvida e, depois, um enunciado que a atenda. Cada ciclo de sequenciamento começa situando o aprendiz sobre o estado atual dele frente ao conhecimento do domínio. 
Agora, que a perícia inicial já foi desenvolvida, a ferramenta provê uma lista de prioridades com sugestões de perícias. A ordenação heurística ponderou critérios como a fronteira do Modelo do Aprendiz e o restante do conhecimento do domínio, a distância dessas perícias até aquela definida como inicial e, por fim, as estratégias de tutoria bemsucedidas para o perfil em particular. Detalhes sobre essa ponderação podem ser consultados em [Moreira 2016].

Apresentam-se as sugestões de perícias para escolha do aprendiz. A ferramenta informa, ao lado do identificador de cada perícia sugerida, a distância dela até a perícia inicial e a prioridade definida, respectivamente. A prioridade de uma perícia considera a citada distância e a ponderação das relações genéticas que a conectam ao modelo do Aprendiz (sendo que, quanto menor o número, maior a prioridade). No momento da seleção, além de cada perícia sugerida ser destacada no conhecimento do domínio, também se indica a estratégia de tutoria mais promissora para desenvolvê-la, conforme o perfil do aprendiz. As seguintes sugestões de perícias são propostas ao aprendiz, adicionadas das informações recém-descritas:

1. variáveis $(3,20)$ : pré-requisito;

2. atribuição $(3,24)$ : analogia;

3. entrada $(3,24)$ : analogia; e

4. entrada $\times$ saída $(4,38)$ : generalização.

Assume-se a primeira sugestão, variáveis, tenha sido selecionada. Logo, deve-se procurar por enunciados que contemplem o reconhecimento de variáveis na análise e na resolução de problemas. Diante do catálogo, tem-se as sugestões abaixo, relacionadas com a quantidade de perícias não desenvolvidas e o total de perícias exercitadas:

1. Olá usuário $(5,11)$;

2. Metade de um real $(6,12)$; $\mathrm{e}$

3. Gastos em viagem $(8,13)$.

A primeira sugestão, Olá usuário, foi aceita pelo aprendiz. Ela abrange uma menor quantidade de perícias não desenvolvidas e, por isso, encontra-se mais próxima do seu estágio atual de conhecimento (Zona de Desenvolvimento Proximal). Procedendo-se corretamente com a resolução do exercício, o Modelo do Aprendiz é atualizado a fim de registrar que as perícias exercitadas foram desenvolvidas.

Possíveis alternativas que tratem de remediação de erros foram desconsideradas na pesquisa. Caso necessário, pode ser escolhido outro enunciado para desenvolver a mesma perícia em uma nova abordagem. Outra alternativa possível seria retroceder às sugestões de perícias e optar por desenvolver outra delas. A ferramenta prossegue avaliando a resolução do aprendiz, atualizando o seu modelo, sugerindo perícias a serem desenvolvidas e enunciados correspondentes.

\section{Estudo Empírico}

O estudo empírico relatado nesta sessão observa a aderência didática do protótipo, tendo em vista a comparação das sugestões fornecidas pelo processo de sequenciamento com outras dadas por tutores humanos. Considerou-se, especialmente, a proximidade entre os arranjos e a ocorrência de inversões severas, na ordem dos tópicos e dos enunciados sugeridos. 
V Congresso Brasileiro de Informática na Educação (CBIE 2016)

Anais dos Workshops do V Congresso Brasileiro de Informática na Educação (CBIE 2016)

\subsection{Sujeitos}

Foram sujeitos da pesquisa três docentes ${ }^{1}$ da área de Ciência da Computação, todos doutores, que ministram disciplinas introdutórias de Programação de Computadores. Nenhum dos docentes teve envolvimento anterior com a pesquisa.

\subsection{Instrumentos}

Um questionário com três questões foi utilizado como instrumento para coleta de dados dos docentes. Adicionalmente, foram fornecidos os seguintes materiais, entregues impressos aos docentes:

1. Um texto de apresentação resumindo a pesquisa;

2. O conhecimento do domínio representado em grafo genético, plotado; e

3. O catálogo de enunciados, diagramado no formato de cartões em tamanho A5. Cada cartão continha um enunciado e a região abrangida do grafo genético.

\subsection{Questionário}

Os questionário entregue aos docentes foi reproduzido integralmente na sequência:

\section{Questão 1}

Ordene os enunciados do catálogo na sequência didática que você considere mais conveniente. Entregue os cartões nessa ordem.

\section{Questão 2}

Considere que o aprendiz domina o conceito de algoritmo, conhece os tipos primitivos de dados, variáveis, constantes, bem como as instruções de entrada, de saída e de atribuição. Diante do catálogo de enunciados representado pelos cartões, qual seria o próximo enunciado que você recomendaria? Justifique.

\section{Questão 3}

Considere o estágio de desenvolvimento do aprendiz ilustrado pela Figura 4. Diante do catálogo de enunciados representado pelos cartões, qual seria o próximo enunciado que você recomendaria? Justifique.

\subsection{Procedimentos}

Detalha-se, a seguir, as orientações fornecidas e o propósito de cada uma das questões componentes. Para comparação, foram realizadas simulações no protótipo em resposta às mesmas questões perguntadas aos docentes. Como procedimento, assumiu-se sempre a primeira sugestão de perícia e de enunciado oferecida pela ferramenta.

\section{Questão 1}

Procura saber como os docentes ordenariam didaticamente o mesmo catálogo de enunciados utilizado pela pesquisa. Os cartões foram entregues embaralhados aos docentes, que ficaram livres para considerar a representação em grafo de cada enunciado.

\footnotetext{
${ }^{1}$ Coordenação do Curso de Tecnologia em Sistemas para Internet, da Universidade Tecnológica Federal do Paraná (UTFPR), Câmpus Guarapuava.
} 
V Congresso Brasileiro de Informática na Educação (CBIE 2016)

Anais dos Workshops do V Congresso Brasileiro de Informática na Educação (CBIE 2016)

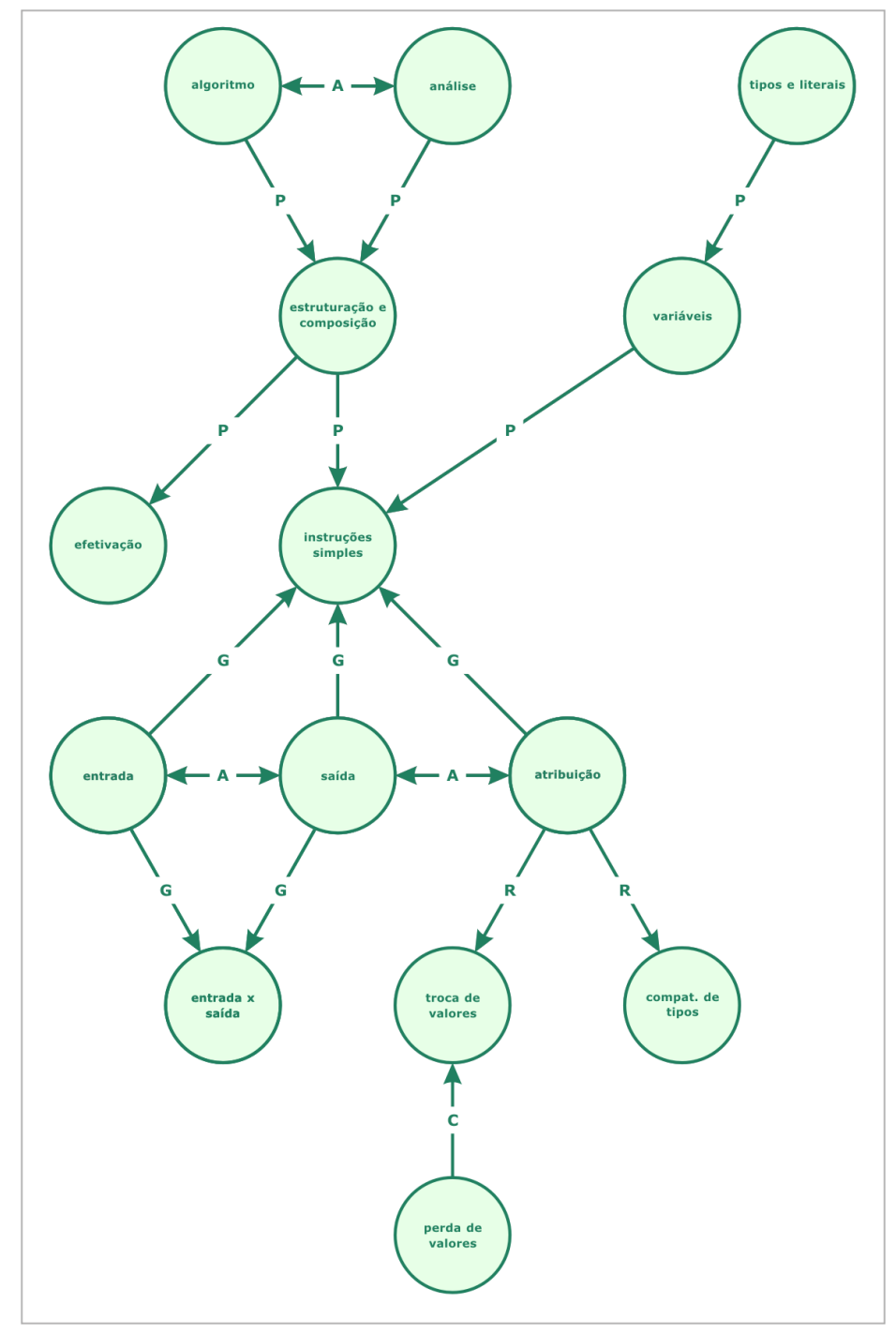

Figura 4. Questão 3

\section{Questão 2}

Constrói um cenário do conhecimento atual do aprendiz para que o docente recomende o próximo enunciado, dentre aqueles do catálogo. Não faz referência à

\section{Questão 3} representação em grafo.

Em contraste com a questão anterior, remete-se diretamente ao grafo genético e induz que o docente decida com base nas mesmas informações assumidas pelo protótipo, dado um cenário para que um enunciado seja sugerido.

\section{Resultados Coletados}

Os resultados foram coletados por meio dos instrumentos de observação especificados na Seção 4.2. São interpretados conforme segue.

\subsection{Questão 1}

A ordenação do catálogo de enunciados pelos docentes consta na Tabela 1. Na análise dessas informações, primeiramente foi observado se houve alguma inversão de conteúdos. 
V Congresso Brasileiro de Informática na Educação (CBIE 2016)

Anais dos Workshops do V Congresso Brasileiro de Informática na Educação (CBIE 2016)

Pode-se perceber isso porque o catálogo contempla os seguintes conteúdos:

1. Instruções de entrada, de saída e de atribuição;

2. Estruturas de condicionais; e

3. Estruturas de repetição.

\begin{tabular}{|c|c|c|c|}
\hline Ordem & Professor 1 & Professor 2 & Professor 3 \\
\hline 1 & Olá mundo & Olá mundo & Olá mundo \\
\hline 2 & Olá usuário & Olá usuário & Olá usuário \\
\hline 3 & Metade de um real & Metade de um real & Troca de valores \\
\hline 4 & Troca de valores & Gastos em viagem & Metade de um real \\
\hline 5 & Média final & Média final & Média final \\
\hline 6 & Conta bancária & Conversão para binário & Gasto com viagem \\
\hline 7 & Gasto em viagem & Conta bancária & Número espelhado \\
\hline 8 & Caixa eletrônico & Número espelhado & Conversão para binário \\
\hline 9 & Número espelhado & Caixa eletrônico & Conta bancária \\
\hline 10 & Conversão para binário & Troca de valores & Caixa eletrônico \\
\hline 11 & Valor absoluto & Valor absoluto & Par ou ímpar \\
\hline 12 & Par ou ímpar & Par ou ímpar & Positivo, negativo ou nulo \\
\hline 13 & Horário válido & Vogais & Valor absoluto \\
\hline 14 & Aprovado ou reprovado & Idade & Maior de três valores \\
\hline 15 & Menor de três valores & Horário válido & Menor de três valores \\
\hline 16 & Maior de dois valores & Aprovado ou reprovado & Horário válido \\
\hline 17 & Positivo, negativo ou nulo & Maior de dois valores & Vogais \\
\hline 18 & Vogais & Menor de três valores & Aprovado ou reprovado \\
\hline 19 & Tipos de triângulo & Positivo, negativo ou nulo & Tipos de triângulo \\
\hline 20 & Idade & Tipos de triângulos & Idade \\
\hline 21 & Colar na prova & Colar na prova & Colar na prova \\
\hline 22 & Maioridade & De 1 até 100 & De 50 até 200 \\
\hline 23 & Soma dos números & De 50 até 200 & De 1 até 100 \\
\hline 24 & De 50 até 200 & Soma dos números & Tabuada do 5 \\
\hline 25 & De 1 até 100 & Tabuada do 5 & Tabuadas do 1 ao 10 \\
\hline 26 & Tabuada do 5 & Potenciação & $1 / \mathrm{n}$ \\
\hline 27 & Tabuadas do 1 ao 10 & Fatorial & Potenciação \\
\hline 28 & Potenciação & Tabuadas do 1 ao 10 & Fatorial \\
\hline 29 & Fatorial & Maior média & Soma dos números \\
\hline 30 & Menor valor & Número primo & Maioridade \\
\hline 31 & Pares e ímpares & Primos após o 100 & Pares e ímpares \\
\hline 32 & Menor e maior peso & Maioridade & Menor valor \\
\hline 33 & Validação & Pares e ímpares & Menor e maior peso \\
\hline 34 & Calculadora & Menor valor & Média dos pares \\
\hline 35 & Média dos pares & Menor e maior peso & Validação \\
\hline 36 & Maior média & Calculadora & Calculadora \\
\hline 37 & Fibonacci & Validação & Número primo \\
\hline 38 & $1 / \mathrm{n}$ & Média dos pares & Primos após o 100 \\
\hline 39 & Número primo & Fibonacci & Maior média \\
\hline 40 & Primos após o 100 & $1 / \mathrm{n}$ & Fibonacci \\
\hline
\end{tabular}

Tabela 1. Resultados dos docentes para a Questão 1

Nesses termos, foi examinado se algum enunciado que contemplasse conteúdo posterior foi antecipado. Ou ainda, se ocorreu o contrário, de um enunciado que requereria 
V Congresso Brasileiro de Informática na Educação (CBIE 2016)

Anais dos Workshops do V Congresso Brasileiro de Informática na Educação (CBIE 2016)

conteúdo mais básico ser prorrogado. Esses casos não aconteceram e a mesma tabela foi seccionada em três partes, em correspondência respectiva aos conteúdos citados.

Depois, constatou-se que o sequenciamento dos enunciados que contemplam um determinado conteúdo é bastante sensível à didática do docente. Assim, por exemplo, o enunciado Vogais, que remete ao conceito de estrutura condicional com seleção múltipla, variou bastante na sequência, porque o Professor 1 preferiu postergar esse conteúdo, enquanto os outros dois decidiram antecipá-lo.

A proposta desta questão aos docentes desempenhou um papel importante na pesquisa. Ela indicou a necessidade de alterações na primeira versão do protótipo porque, em algumas situações, havia antecipação significativa de conteúdos. Foi necessário reexaminar sobre como os enunciados estavam anteriormente elicitados na ferramenta.

A última simulação feita com o protótipo, apresentada pela Tabela 2, forneceu um sequenciamento próximo ao dos docentes e também não trouxe inversão entre os três conteúdos. O sequenciamento sugerido possui menos enunciados porque, conforme mencionado, a configuração atual do protótipo assume que a resolução correta de um único enunciado já proporciona o desenvolvimento da perícia contemplada. Assim, não são repetidos enunciados que abranjam unicamente perícias já desenvolvidas.

\begin{tabular}{|c|l|}
\hline Ordem & Protótipo \\
\hline 1 & Olá mundo \\
\hline 2 & Olá usuário \\
\hline 3 & Metade de um real \\
\hline 4 & Reajuste de preço \\
\hline 5 & Troca de valores \\
\hline 6 & Gastos com viagem \\
\hline 7 & Valor absoluto \\
\hline 8 & Horário válido \\
\hline 9 & Vogais \\
\hline 10 & Idade \\
\hline 11 & Menor de três valores \\
\hline 12 & Colar na prova \\
\hline 13 & Validação \\
\hline 14 & Tabuadas do 1 ao 10 \\
\hline 15 & Número primo \\
\hline
\end{tabular}

Tabela 2. Resultados do protótipo para a Questão 1

\subsection{Questão 2}

Os três docentes sugeriram um enunciado que introduzisse estruturas condicionais ao aprendiz, a saber:

- Professor 1: Valor absoluto;

- Professor 2: Valor absoluto;

- Professor 3: Par ou ímpar.

As escolhas dos docentes corroboraram a ordenação de enunciados que foi recomendada na questão anterior, uma vez que a sugestão de cada um coincide com o primeiro enunciado que trata o referido conteúdo naquela ordenação (Tabela 1). O protótipo alcançou o mesmo comportamento de dois docentes, sugerindo o enunciado Valor absoluto. 
V Congresso Brasileiro de Informática na Educação (CBIE 2016)

Anais dos Workshops do V Congresso Brasileiro de Informática na Educação (CBIE 2016)

\subsection{Questão 3}

Diante do conhecimento do domínio, os docentes tinham três opções de perícias:

- Constantes;

- Variáveis $\times$ constantes;

- Expressões aritméticas.

Entretanto, o catálogo não possui enunciado que contemple perícias relacionadas a constantes. Essa situação foi propositalmente avaliada para elaborar o questionário.

Foram sugestões dos docentes:

- Professor 1: Média final, por contemplar a perícia expressões aritméticas;

- Professor 2: a autoria de um enunciado que introduzisse a perícia constantes;

- Professor 3: Metade de um real, por contemplar a perícia expressões aritméticas.

O protótipo sugere a perícia constantes e, no próximo passo, não recomenda enunciados, indicando a necessidade de autoria. Ele permite retroceder e optar pela perícia expressões aritméticas, para então sugerir o enunciado Metade de um real.

\section{Considerações Finais e Trabalhos Futuros}

Diante do exposto, a solução proposta obteve resultados bastante próximos àqueles fornecidos por tutores humanos. Destaca-se que não houve inversões de ordem significativas nas sugestões de perícias ou de enunciados feitas pelo protótipo. Além disso, foi notado que a ordem dos enunciados diferiu mesmo entre os docentes sujeitos da pesquisa, entendo-se ser sensível, em certo grau, à didática de cada tutor humano em particular. A solução, por sua vez, acaba sendo influenciada pela perspectiva com que se elicitam os enunciados, no momento da catalogação, quanto às perícias contempladas (regiões do grafo).

Assim, acredita-se que informações interessantes possam provir de experimentos adicionais. Algumas possibilidades, ainda na comparação com tutores humanos, residem na condução de dois outros experimentos distintos, um excluindo os detalhes da metodologia (tais como terminologia e representação) e outro imergindo os docentes nessas informações. Os resultados seriam utilizados para adequação dos parâmetros e aprimoramento da metodologia proposta, bem como para uma investigação mais profunda do problema.

\section{Referências}

Goldstein, I. P. (1979). The genetic graph: A representation for the evolution of procedural knowledge. International Journal of Man-Machine Studies, 11(1):51-77.

Maschio, E. (2013). Modelagem do Processo de Aquisição de Conhecimento Apoiado por Ambientes Inteligentes. Tese de doutorado, Universidade Federal do Paraná, Curitiba.

Miliszewska, I. and Tan, G. (2007). Befriending Computer Programming: A Proposed Approach to Teaching Introductory Programming, volume 4, pages 277-289. Issues in Informing Science and Information Technology.

Moreira, C. (2016). Sequenciamento Inteligente e Adaptativo de Enunciados em Programação de Computadores. Dissertação de mestrado, Universidade Federal do Paraná, Curitiba. 
V Congresso Brasileiro de Informática na Educação (CBIE 2016)

Anais dos Workshops do V Congresso Brasileiro de Informática na Educação (CBIE 2016)

Moreira, C., Pimentel, A. R., and Maschio, E. (2015). Estudo sobre o sequenciamento inteligente e adaptativo de enunciados em programação de computadores. Anais dos Workshops do IV Congresso Brasileiro de Informática na Educação (CBIE), pages 1320-1329.

Norvig, P. (2001). Teach yourself programming in ten years. http://norvig.com/21days.html.

Nwana, H. S. (1990). Intelligent tutoring systems: an overview. Artificial Intelligence Review, (4):251-277.

Pimentel, A. R. and Direne, A. I. (1998). Medidas cognitivas no ensino de programação de computadores com sistemas tutores inteligentes. In Anais do IX Simpósio Brasileiro de Informática na Educação. SBIE 1998, volume 9, pages 206-215, Fortaleza, CE, Brasil. Sociedade Brasileira de Computação.

Weber, G. and Brusilovsky, P. (2001). ELM-ART: An adaptive versatile system for webbased instruction. In International Journal of Artificial Intelligence in Education, volume 12, pages 351-384.

Weber, G. and Mollenberg, A. (1995). Cognition and Computer Programming, chapter 14 - ELM programming environment: A tutoring system for LISP beginners, pages 373-408. Ablex Publishing Corporation, Norwood, New Jersey. 